\title{
Simulating individual word identification thresholds and errors in the fragmentation task
}

\author{
JOHANNES C. ZIEGLER and ARNAUD REY \\ Center for Research in Cognitive Neuroscience, CNRS, Marseille, France \\ and \\ ARTHUR M. JACOBS \\ Center for Research in Cognitive Neuroscience, CNRS, Marseille, France \\ and Philipps-University, Marburg, Germany
}

\begin{abstract}
This article presents a large-scale study that collected word identification thresholds and errors in the fragmentation task for all four-letter French words. In the first part of this article, we identify some of the variables (e.g., word frequency, neighborhood size, letter confusability) that affect performance in the fragmentation task. In the second part, we analyze individual response performance and identify different response strategies. We demonstrate that the interactive activation model can account for individual response strategies by adapting two of its original parameters: word-letter feedback and letterword inhibition. In the third part, we demonstrate that the adaptation of the interactive activation model to the fragmentation task makes it possible to successfully simulate a facilitatory frequency effect on identification thresholds, an inhibitory neighborhood size effect on error rates, and an inhibitory letter confusability effect on identification thresholds. When the task-specific processes of the fragmentation task are specified and individual response strategies are considered, the interactive activation model provides a parsimonious architecture for modeling the task-independent processes involved in word perception.
\end{abstract}

Laboratory studies may only discover contextually situated performance - the fluid coupling of subject and laboratory method - the complex interface of organism and environment.

-G. C. Van Orden, personal communication, November 12, 1995

Reading is typically studied by manipulating a set of perceptual and linguistic variables within a variety of laboratory methods (tasks). Each task is believed to tap into a common mental structure that is involved in reading. However, each task also contains some idiosyncratic, task-

This research was partially supported by a grant from the German Academic Exchange Service (DAAD-Doktorandenstipendium aus Mitteln des zweiten Hochschulsonderprogramms) to J.Z., a postdoctoral fellowship from the Fondation Fyssen to J.Z., Grant 95124 from the French Ministry of Education and Research (programme Sciences Cognitives) to A.R., and a grant from the "Deutsche Forschungsgemeinschaft (DFG)" to A.J. (Teilprojekt 7 der Forschergruppe "Dynamik kogitiver Repräsentationen"). We are grateful to Sylvan Kornblum for extremely helpful comments on a previous version of this article. Many thanks also to Jonathan Grainger, Patrick Lemaire, Geoffrey Loftus, Marie Montant, Gay Snodgrass, and various anonymous reviewers for their invaluable critical feedback. We are grateful to Monique Chiambretto for technical assistance. Correspondence concerning this article should be addressed to J. C. Ziegler, Centre de Recherche en Neurosciences Cognitives, Centre National de la Recherche Scientifique, 31, Chemin Joseph Aiguier, 13402 Marseille Cedex 20, France (e-mail: ziegler@lnf.cnrs-mrs.fr).

-Accepted by previous editor, Geoffrey R. Loftus specific requirements that reveal nothing, or only a little, about the common mental structure underlying reading. This scenario contains at least three challenges for cognitive psychology. The first challenge is to tease apart the task-specific aspects from those aspects that may truly reflect the contents, structure, and functioning of the cognitive system. The second challenge is to capture the flexibility and variability with which subjects seem to adapt to a particular task - that is, the fluid coupling of subject and task mentioned above. The third challenge involves finding and manipulating a set of perceptual and linguistic variables that helps us to identify the content, structure, and processes of the reading system.

\section{What Is Task-Specific and What Is Not?}

The first challenge requires, on the one hand, that one specify and implement what one believes to be the common cognitive architecture underlying performance across a number of different tasks. On the other hand, it requires that one specify and implement those properties that are specific to a particular task. For example, perceptual identification, lexical decision, and letter search may all tap into a common cognitive structure that is involved in reading. However, they all clearly involve some task-specific mechanisms, such as identifying words when they are briefly presented and masked, discriminating words from nonsense letter strings, or deciding whether a word contains a particular letter. Therefore, the modeling of performance in a particular task requires that one specify the task-specific read-out mechanisms, the connection be- 
tween the processes within the model, and the observed dependent variables.

Recently, Grainger and Jacobs (1993, 1994, 1996; Jacobs \& Grainger, 1992) pursued such a modeling approach for the lexical decision, the perceptual identification, and the Reicher tasks. They chose the implemented and wellspecified interactive activation model (McClelland \& Rumelhart, 1981; Rumelhart \& McClelland, 1982) as the common architecture underlying word perception in different tasks. In addition, they implemented a number of task-specific mechanisms (read-out procedures) that allowed them to predict percentage of errors, reaction time (RT) means and distributions, and strategic influences in specific tasks. For example, they suggested that three processes underlie a speeded binary response in the lexical decision task: Two of the processes use lexical information to generate a yes response, and the third uses both lexical and time information to generate a no response. These sources of information are: (1) the overall (global) activity in the orthographic lexicon, operationalized in the simulation model as the sum of the activation levels of all word units; (2) the (local) activity of word units in the orthographic lexicon, operationalized as the activation level of individual word units; and (3) the time (cycles) from stimulus onset.

In the present paper, we generalize Grainger and Jacobs's (1996) approach by investigating and modeling performance in a different task: the screen fragmentation procedure (Snodgrass \& Mintzer, 1993; Snodgrass \& Poster, 1992; Warrington \& Weiskrantz, 1968). According to Snodgrass and Mintzer (1993), this technique presents a "more direct method of measuring visual recognition" (p. 249). In this task, subjects are presented with a highly fragmented word at the beginning of each trial (see Figure 1). They are asked to gradually demask the word until they are able to identify it. This task allows the measurement of identification thresholds and errors. Identification thresholds refer to the level of fragmentation at which the subjects generate a correct response. Errors are responses that do not match the target word in one or more letters.

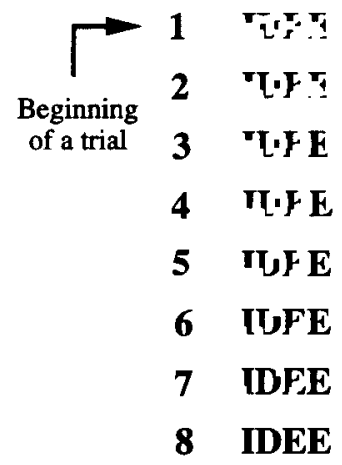

Figure 1. Example of the fragmentation procedure used in the present study. The stimuli (here the French word idée) are first presented at the most fragmented level (Level 1). Subjects gradually demask them until they can identify the words. Note that, in this example, idée may be misidentified as jupe up to Level 4.
With respect to the modeling enterprise described above, simulating performance in the fragmentation task generates a number of questions. Can the interactive activation framework, which is usually thought to reflect the automatic, early, and fast processes involved in word perception, serve as the basic modeling architecture for a task in which subjects can take the time to exploit their lexical and spelling knowledge, develop possible response strategies, and guess correct responses? Can the interactive activation framework be used to simulate error patterns in the fragmentation task? To simulate identification errors, it has to be specified how it happens that, on occasion, incorrect items have higher activation levels than do correct ones and are selected for response. Errors are not only a major variable in the fragmentation task but also in related fields, such as cognitive neuropsychology (see, e.g., Caramazza, 1992) or language production (see, e.g., Dell, 1986; Schade \& Berg, 1992).

In the present article, we add task-specific procedures to the interactive activation model that allow us to predict identification thresholds and errors in the fragmentation task (see the section on the general simulation method). In this respect, the central question is: Can we capture the flexible, strategic, and complex behavior that is observed in the fragmentation task with the basic principles and the simple structure of the interactive activation model?

\section{Individual Predictions}

The second challenge concerns the prediction of individual task performance. A number of cognitive psychologists believe that predicting individual differences and describing strategic adaptation are central issues for theory construction in cognitive psychology (see, e.g., Brown, Lupker, \& Colombo, 1994; Lemaire \& Siegler, 1995; Reder, 1987, 1988; Siegler, 1987; Sperling \& Dosher, 1986; Stone \& Van Orden, 1993; Underwood, 1975). What are the strategies that subjects adopt to maximize performance and minimize the duration of the experiment? How can the interactive activation model predict individual performance and adaptation to task requirements?

The fragmentation task seems to be well suited to pursue these issues since it allows the subjects to trade visual bottom-up information and lexical top-down information by developing an adequate response strategy. For example, subjects may decide to barely demask the stimulus and quickly generate a word identification response by using their lexical and spelling knowledge (guessing strategy). Others may demask the word until they have enough visual information to be sure about its identity (conservative strategy). In this article, we attempt to simulate individual differences and strategic adaptation within the interactive activation framework by systematically manipulating two of the model's original parameters: word-to-letter feedback and letter-to-word inhibition. 


\section{Which Variables Should Be Manipulated?}

The third challenge involves finding and manipulating an interesting, theoretically motivated set of perceptual and linguistic variables that may reveal something about the content, the structure, and the functioning of the reading system. For example, word frequency or the number of a word's orthographic neighbors could be such variables. The problem is that many of the linguistic variables that influence word perception are highly correlated (see, e.g., Frauenfelder, Baayen, Hellwig, \& Schreuder, 1993; Landauer \& Streeter, 1973). This makes it virtually impossible to find two samples of words that differ in the variable(s) of interest and that are matched on all other relevant variables known to influence performance (see Cutler, 1981; Treiman, Mullennix, BijeljacBabic, \& Richmond-Welty, 1995).

As a way out of this dilemma, Treiman et al. (1995) recently proposed that traditional small-scale experiments be supplemented with large-scale studies that are analyzed with regression methods. "In such large-scale studies, there is no attempt to choose stimuli that differ on only the variable(s) of interest and that are matched on 'nuisance' variables. Rather, the characteristics of the stimuli are allowed to vary as they will. Statistical techniques are used to examine the contribution of each variable above and beyond the contribution of all of the other variables" (Treiman et al., 1995, p. 132). This was the approach adopted in the present article (for a similar approach, see Rumelhart \& Siple, 1974; Spieler \& Balota, 1996).

In the present study, the subjects were presented with virtually all four-letter French words in the context of a fragmentation task. Identification thresholds and errors were measured for all of these words. Thus, we did not design one particular experiment with a limited number of stimuli to test for one specific effect (e.g., the frequency effect on identification thresholds), but we collected a large database of identification thresholds and errors for all four-letter French words. This database was analyzed with regression methods; it served as a constraint for developing an interactive activation model for the fragmentation task, one capable of simulating individual identification threshold and error data.

\section{MODEL}

Our modeling approach contains two steps. First, we specify and implement a set of mechanisms and principles that may underlie word perception in different tasks. Second, we implement those mechanisms that may be specific only to the task at hand-here, the fragmentation task. In line with previous simulation work (Grainger \& Jacobs, 1993, 1994, 1996; Jacobs \& Grainger, 1992), we chose McClelland and Rumelhart's (1981) interactive activation model as the common architecture underlying word perception in a variety of reading tasks.

The interactive activation model simply contains a feature level, a letter level, and a word level (orthographic lexicon). Each unit in the feature level represents one of a letter's features, each unit in the letter level represents one letter of the alphabet, and each unit in the word level represents one word of the lexicon. The interactive activation model implements two major principles. The first principle is interactive activation-that is, activation spreads along bidirectional connections between units belonging to different levels. Compatible units have excitatory connections; they mutually activate one another (e.g., the letter unit $L$ in the second letter position activates the word unit blue and vice versa). Incompatible units have inhibitory connections; they mutually inhibit one another (e.g., $L$ in the second position inhibits all word units that do not contain an $L$ in the second position). The second principle is within-level inhibition-that is, units within the same level mutually inhibit one another through inhibitory lateral connections (e.g., each word unit inhibits all other word units).

To simulate performance in the fragmentation task, we had to deal with the following issues. First, the model had to be generalized to include task-specific mechanisms. These mechanisms account for the fact that the visual input in the fragmentation task is degraded during an early level within the sequence of fragmentations. Subjects can gradually add visual information until they are able to identify the word (give a response). Second, some properties of the fragmentation task, such as poor visual input, change the global stability and dynamics of the model. Thus, the standard parameter settings had to be adapted in order to simulate performance in this task. Third, we had to face the possibility that subjects may use different response strategies to optimally perform the task. Fourth, a read-out procedure (i.e., the model's output) had to be implemented that produces identification thresholds and errors (i.e., the dependent variables). The details of the task-specific implementation are given in the section describing the general simulation method.

To summarize, we collected identification thresholds and errors in the fragmentation task for virtually all fourletter French words. Using regression analyses, we determined which perceptual and linguistic variables account for variance in performance in this task. We analyzed individual response strategies. Finally, we propose an extension of the interactive activation model capable of simulating individual identification thresholds and error rates in the fragmentation task.

\section{GENERAL METHOD}

\section{Subjects}

Ten subjects from the Center for Research in Cognitive Neuroscience participated in the experiment. All were native French speakers and had normal or corrected to normal vision. The experiment lasted about $4 \mathrm{~h}$.

\section{Apparatus and Stimuli}

The experiment was controlled by a Compaq 486 personal computer. As experimental stimuli, we used all four-letter French words, excluding those with a frequency of less than 2 occurrences per million, proper names, contractions, abbreviations, and foreign words. This selection resulted in a total of 580 words. Word fre- 
quency ranged from 2 to 25,532 occurrences per million according to a French word frequency count (Imbs, 1971). The words were displayed in uppercase Triplex Vectorial Police (Size 4) of Borland Pascal. Each letter was centered within a $24 \times 32$ pixels square window $(16 \times 32$ pixels square window for thin letters $)$.

\section{Procedure}

The subjects were seated in a dentist chair in a sound-attenuated experimental box. At the beginning of the experiment, the subjects were familiarized with the typography by presenting all the letters of the alphabet. We used the ascending method of limits of the original screen fragmentation procedure, as described by Snodgrass and Poster (1992) and by Snodgrass and Mintzer (1993). During the experimental trials, a test word was first displayed at the most fragmented level of presentation (Level 1). The subjects were asked to gradually demask the word by pressing the space bar of the keyboard until they had sufficient information to generate a response. Identification thresholds and errors were recorded.

This demasking process was entirely controlled by the subjects. As long as they could not identify the word, they were instructed to press the space bar to go on to the next more complete (less fragmented) version of the stimulus. They were asked to give a response by typing the word on the keyboard as soon as they thought that they knew what the stimulus was. The subjects were instructed to respond as soon as possible without making pure guesses. Their response was not corrected. The subjects could give only one response on each trial. Having responded, they could not keep on demasking the word until they found the correct response. Instead, once the response was given, the next trial was automatically initiated. An experimental session was subdivided into 10 blocks of 58 words, and the subjects were invited to make a break between the blocks. The stimuli were presented in a different random order for each subject.

\section{Fragmentation Procedure}

The fragmentation procedure was identical to the one described by Snodgrass and Poster (1992) and by Snodgrass and Mintzer (1993). Words were fragmented in order to produce eight levels of fragmentation (see Figure 1 for an illustration of the eight fragmentation levels). The fragmentation was achieved by randomly deleting blocks of pixels according to the function: $P($ level $)=$ $.85^{(8-\text { level })}$, where $P$ is the proportion of critical pixel blocks retained in the word, and level ranges from 1 (most fragmented) to 8 (complete word). For example, at Level $1,32 \%$ of the pixel blocks were exposed, whereas at Level $4,52 \%$ of the blocks were presented One pixel block consisted of $8 \times 8$ pixels. Fragments were added cumulatively from one level to the next, so that a stimulus at a particular level of fragmentation contained all the features of the stimulus at the preceding level of fragmentation. The words were fragmented on line, with a different random selection of pixel blocks on each word trial.

\section{GENERAL SIMULATION METHOD}

In the interactive activation model, the visual presentation of a letter string initiates the following sequence of events. The visual input activates the corresponding set of feature units. Feature units activate compatible letter units and inhibit incompatible letter units. In turn, each letter unit activates compatible word units, inhibits incompatible word units, and inhibits all other letter units that share the same position. Then, word units feed back activation to all compatible letter units and inhibit all other word units. For each processing cycle, the model proceeds through this same sequence of events. The level of activation of all units is continuously registered. Under normal viewing conditions, the model "recognizes" a word when the activation of its corresponding word unit reaches a critical activation threshold (the $M$-criterion in Grainger \& Jacobs, 1996). Under degraded viewing conditions (as in the fragmentation task), if no unit reaches the activation threshold $M$ before a given time threshold $T$ (i.e., after a given number of processing cycles), then no response is generated and more information is required.

To capture performance in the fragmentation task, the simulation procedure was adapted to mimic the experimental procedure. On each trial, the word was presented to the model in its most fragmented version (Level 1). If a word unit reached the critical activation threshold $M$ before the time threshold $T$, the model made a response. Otherwise, the model made a "pass" and the word was presented at the next level of fragmentation (Level 2). We obtained 10 simulated subjects by presenting the 580 words 10 times to the model. The simulation of individual identification thresholds and errors in the fragmentation task required modifications in the input procedure, the parameter settings, and the read-out procedure. These will be described next.

\section{Input Procedure}

The fragmented input for the model was obtained by randomly activating a certain number of feature detectors at the feature level. The number of activated features equaled the proportion $P$ of pixel blocks that were used to obtain the stimulus fragmentation in the experiment. For example, at fragmentation Level $1,32 \%$ of the features were randomly selected and activated in the model. Note that, whereas McClelland and Rumelhart (1981) used 14 features to represent all letters, we used a more detailed matrix of 43 features to represent each letter. We increased the number of features in order to better capture particularities of the typography and similarities (confusability) between letters.

\section{Parameter Settings}

The model contains excitatory (alpha) and inhibitory (gamma) connections. The impact (weight) of each type of connection is determined by a parameter. Essentially, there is one set of parameters governing the flow of activation and inhibition between the feature and the letter level, one between the letter and the word level, and one within each level. These parameters crucially constrain the global stability and dynamics of the model. In the following section, we describe how these parameters were adapted in order to simulate performance in the fragmentation task.

1. Feature-letter connections. In the original interactive activation model (McClelland \& Rumelhart, 1981), the absence of a feature was considered as informative as its presence. For example, if subjects recognize a visual configuration that looks like the letter $F$, the absence of the bottom line will tell them that it cannot be the letter $E$. Therefore, in the original model, both the presence and the absence of a feature determine letter activation. For example, on the one hand, if the bottom line (_) is present in a given stimulus, the corresponding feature unit sends excitation to all letter units that contain this feature (e.g., $E, L$ ) and inhibition to all letter units that do not contain this feature (e.g., $F, V$ ). On the other hand, if the bottom line is absent, this absent-feature unit sends excitation to all letter units that do not contain this feature (e.g., $F, V$ ) and inhibition to all letters that do contain this feature (e.g., $E, L$ ). The original parameter values for these excitatory and inhibitory connections between the feature and the letter levels were .005 and .15 , respectively.

In the fragmentation task, however, the absence of a feature is less informative than its presence. At an early level of fragmentation, a feature may simply be absent as a consequence of fragmentation and not because it is truly absent in the letter. For example, if subjects recognize a visual configuration in a highly fragmented stimulus that looks like the letter $F$, the absence of the bottom line will not guarantee that the letter is an $F$; it could be the letter $E$, in which the bottom line is missing because of fragmentation. To accommodate for those task-specific aspects, the connection weights of absent features were changed as a function of the level of fragmentation. Excitatory connections between absent features and letter units were set to become stronger as the stimulus gets more visible 
$(\alpha=.005 * P$, where $P$ varies between .32 and 1.0 and represents the proportion of displayed pixel blocks; see the general method section). Similarly, inhibitory connections between absent features and letter units were set to increase exponentially as the stimulus gets more visible $[\gamma=.15 * \exp (190 *((P / 10)-.1))]$. This modification guarantees that absent features have nearly no impact on letter activation when the stimulus is highly fragmented; they become more important as stimulus information increases, and they reach their original parameter value (i.e., .005 and .15, respectively) when the complete word is displayed (i.e., $P=1.0$ ).

2. Letter-letter and word-word connections. Letter units within the letter level and word units within the word level mutually inhibit each other. This lateral inhibition principle implements a competition process between units belonging to the same processing level. The parameters governing the flow of inhibition through the lateral connections were set at .04 within the letter level and at .21 within the word level.

3. Letter-word excitatory connections. Letter units activate word units that are compatible with them. The parameter governing this flow of activation was set at .07 .

4. Letter-word inhibitory and word-letter excitatory connections. The values of the parameters governing the flow of inhibition between letter and word units and the flow of activation (feedback) between word and letter units were determined for each subject; they varied as a function of a subject's individual performance and strategic adaptation to the task requirements (a point that will be further developed in the results section). An increase in letter-word inhibition can be interpreted as an increase in a subject's ability and effort in paying attention to the letter information. The more he or she focuses on the bottom-up letter information, the better he or she is at inhibiting incompatible word candidates. In contrast, an increase in word-letter excitation (feedback) can be interpreted as an increase in a subject's ability to use lexical information to mentally fill in missing fragments. The exact values for these parameters are given in the results section.

\section{Read-out Procedure}

The model made a response when a word unit reached the response threshold $(M)$ before the time threshold $(T)$. If no word unit reached the $M$ threshold before the $T$ threshold, the model passed on to the next less fragmented level (i.e., more feature detectors were activated, in accordance with the input procedure described above). The cycle at which a word unit reaches the $M$ threshold corresponds to the model's identification threshold. If a word unit that is different from the input stimulus reaches the activation threshold $M$, the model has incorrectly identified a word: it made an error.

As in previous simulation work (see, e.g., Grainger \& Jacobs, 1996; Jacobs \& Grainger, 1992), we used normally distributed $M$ and $T$ thresholds. The mean of the distribution for the $M$ threshold was .67 with an $S D$ of .09 (this distribution was truncated at .90 for the upper value and .15 for the lower value). The mean of the distribution for the $T$ threshold was 22 processing cycles with an $S D$ of 3 (this distribution was truncated at 28 for the upper value and 17 for the lower value).

\section{RESULTS AND DISCUSSION}

The results section is organized into three parts. First, we present results from a multiple regression analysis that uncovers which of the independent variables account for a significant portion of the variance in the identification threshold and error data of the fragmentation task. Second, we analyze individual response patterns and demonstrate how a variant of the interactive activation model can account for such response patterns. Third, we evaluate the model's ability to simulate the subjects' per- formance with respect to those variables and effects that account for the major part of the variance in the regression analysis.

\section{Regression Analysis}

The two performance measures (dependent variables) in the fragmentation task were identification thresholds for correct word identifications and percentage of errors. The subject's identification threshold for each word was defined as the level of fragmentation at which correct identification occurred. Identification thresholds varied between 1 and 8 . The percentage of errors corresponded to the percentage of responses that did not match the target word in one or more letters (incorrect word identifications).

As independent variables, we chose a number of variables that had been shown to influence visual word recognition performance in a variety of word recognition paradigms. These variables were plain and logarithmic word frequency ( $\mathrm{F}$ and $\log \mathrm{F}$, respectively), neighborhood size $(\mathrm{N})$, the number of higher frequency neighbors (HFN), summed positional letter frequency (LF), summed positional bigram frequency (BF), and letter confusability in the fragmentation task (CONF).

To briefly characterize these variables, word frequency is known to systematically facilitate performance in lexical decision, naming, and perceptual identification (see, e.g., Forster \& Chambers, 1973; for a review, see Monsell, 1991). Neighborhood size is defined as the number of words that can be obtained by changing one letter of the word (Coltheart, Davelaar, Jonasson, \& Besner, 1977). Neighborhood size and the number of higher frequency neighbors have been found to facilitate naming performance (Peereman \& Content, 1995), inhibit perceptual identification performance (Grainger \& Jacobs, 1996; Grainger, O’Regan, Jacobs, \& Segui, 1989, 1992; Snodgrass \& Mintzer, 1993), and inhibit and/or facilitate lexical decision performance, depending on the type of nonwords, list composition, and the strategy of the subjects (Andrews, 1989, 1992; Huntsman \& Lima, 1996; Sears, Hino, \& Lupker, 1995; for a review, see Grainger \& Jacobs, 1996). Positional letter and bigram frequency are measures of orthographic redundancy. Orthographic redundancy might help or hurt performance, depending on the task requirements (see Grainger \& Jacobs, 1993; Massaro \& Cohen, 1994; Massaro, Jastrzembski, \& Lucas, 1981). The confusability index is a graded empirical measure that was derived from a previous study (Rey, Ziegler, Montant, \& Jacobs, 1996). In this study, we obtained mean correct identification thresholds for all letters of the alphabet in exactly the same way as we did for the words in the present study. These mean identification thresholds served as an index of letter confusability. Accordingly, the confusability index in the present study was defined as the sum of the mean identification thresholds of a word's constituent letters. This confusability index was normalized to vary between 0 and 1 .

A total of 580 cases (mean values for all words averaged across subjects) went into the regression analysis. Simple correlations for the two dependent measures (identi- 
fication thresholds and errors) and the seven independent measures were calculated. Partial correlations, reflecting the correlation of each independent variable with the variance of all other variables pulled out, were carried out on each of the dependent measures. Table 1 gives the simple and partial correlations and their corresponding significance level.

The analysis of the simple correlations makes it possible to address three questions: (1) What are the variables that are most strongly correlated with performance in the fragmentation task? (2) Does a variable that accounts for variance in the threshold data also account for variance in the error data? (3) Are the independent measures intercorrelated and, if so, to which extent?

As concerns the first question, the threshold data are most strongly correlated with logarithmic word frequency and letter confusability: the lower the frequency of a word, the higher its identification threshold; the higher the confusability of a word's constituent letters, the higher its identification threshold. The error data are most strongly correlated with the neighborhood measures ( $N$ and $H F N$ ), letter frequency, and letter confusability: Error rates increase as a function of the density and frequency of a word's orthographic neighborhood, its letter frequency, and the degree of its confusability.

As concerns the second question, there seems to be an interesting dissociation: Although word frequency is strongly correlated with identification thresholds, it is not significantly correlated with error rates. In contrast, although the neighborhood variables (N and HFN) and letter frequency are strongly correlated with error rates, they are not significantly correlated with identification thresholds. Only the confusability index is strongly correlated with both variables; however, it is more strongly correlated with identification thresholds than with error rates.

As concerns the third question, it can clearly be seen in Table 1 that most of the independent measures are highly intercorrelated. For example, logarithmic word frequency is highly correlated with letter and bigram frequency $(.193$ and .432 , respectively). Neighborhood size is highly cor- related with the number of higher frequency neighbors, letter frequency, and bigram frequency $(.721, .546$, and .313 , respectively). Only the confusability index does not seem to be significantly correlated with any of the other independent variables.

The analysis of the partial correlations makes it possible to examine the contribution of each variable above and beyond the contribution of all other variables. This analysis confirmed that logarithmic word frequency and confusability accounted for some unique variance on identification thresholds ( -.313 and .449 , respectively), whereas neighborhood size, letter frequency, and confusability accounted for unique variance on error rates (.164, .151 , and .160 , respectively). Bigram frequency functioned as a classic suppressor variable: It was not correlated with the dependent measures (thresholds, errors), but it was strongly correlated with other independent variables (e.g., $\mathrm{F}, \mathrm{N}$, and LF). This way, it bound some of the irrelevant variance of the other independent variables, which resulted in its significant partial correlation. To summarize, it seems that identification threshold data can be accounted for on the basis of word frequency and confusability; error data seem to be best accounted for by neighborhood structure, orthographic redundancy, and confusability.

\section{Individual Predictions}

The following analyses address the question as to whether there are individual differences in how subjects perform the fragmentation task. ${ }^{1}$ If so, can the interactive activation model be tuned to predict individual performance? In order to identify individual response strategies, we plotted the mean correct identification threshold for each subject against its mean error rate (averaged across the 580 words). These data are presented in Figure 2A.

Figure $2 \mathrm{~A}$ clearly reveals individual response patterns in the fragmentation task. The performances of Subjects 1-7 reflect a speed-accuracy tradeoff-that is, subjects trade levels (speed) against errors (accuracy). For example, Subjects $1-4$ responded late and made only a few errors (conservative response strategy). In contrast, Sub-

Table 1

Simple and Partial Correlations Between Identification Thresholds (Levels) and Errors and Seven Independent Variables

\begin{tabular}{|c|c|c|c|c|c|c|c|c|c|c|c|}
\hline \multirow[b]{2}{*}{ Variable } & \multicolumn{9}{|c|}{ Simple } & \multicolumn{2}{|c|}{ Partial } \\
\hline & Levels & Errors & $\mathrm{F}$ & $\log F$ & $\mathrm{~N}$ & HFN & LF & $\mathrm{BF}$ & Conf & Levels & Errors \\
\hline Levels & & & & & & & & & & & \\
\hline Errors & $.192 \dagger$ & & & & & & & & & & \\
\hline $\mathrm{F}$ & -.052 & -.060 & & & & & & & & .024 & -.018 \\
\hline $\log F$ & $-.224 \uparrow$ & -.039 & $.475 \dagger$ & & & & & & & $-.313 \dagger$ & -.033 \\
\hline $\mathrm{N}$ & -.047 & $.310^{\dagger}$ & -.031 & .016 & & & & & & -.016 & $.164 \dagger$ \\
\hline HFN & .035 & $.223 \dagger$ & $-.130^{*}$ & $-.376 \dagger$ & $.721 \dagger$ & & & & & -.059 & -.025 \\
\hline $\mathrm{LF}$ & -.024 & $.240 \dagger$ & $.193 \dagger$ & $.143 \dagger$ & $.546 \dagger$ & $.382 \dagger$ & & & & -.004 & $.151 \dagger$ \\
\hline $\mathrm{BF}$ & .027 & .046 & $.432 \uparrow$ & $.293 \dagger$ & $.313+$ & $.196 \dagger$ & $.593 \dagger$ & & & $.138 \dagger$ & $-.092 *$ \\
\hline Conf & $.427+$ & $.146 \dagger$ & -.028 & -.009 & -.004 & -.008 & -.068 & -.066 & & $.449 \dagger$ & $.160 \dagger$ \\
\hline
\end{tabular}

Note-F, frequency; N, number of neighbors; HFN, number of higher frequency neighbors; LF, letter frequency; BF, bigram frequency; Conf, confusability index. ${ }^{*} p<.05 .{ }^{\dagger} p<.001$. 

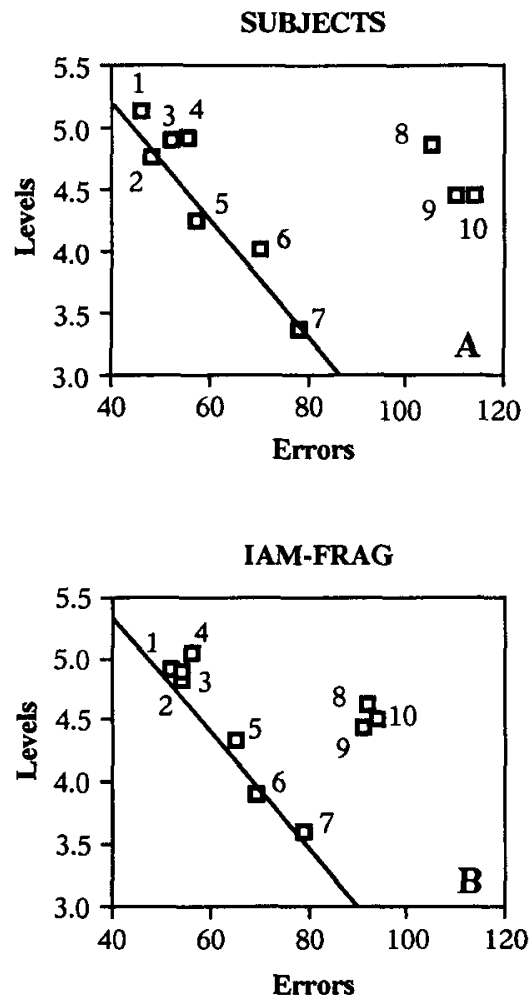

Figure 2. Individual response patterns for the subjects (panel A) and the model (panel B). The numbers next to the data points indicate the numbers of real and simulated subjects.

ject 7 responded earlier and made more errors (guessing strategy). Subjects 5 and 6 lie in between these two extremes.

However, the performance of Subjects 8-10 does not fall on the regression line that describes the speedaccuracy tradeoff for Subjects 1-7. Subjects 8-10 responded rather late and committed a large number of errors. An analysis of their individual response patterns suggests a particular type of response strategy. These subjects seem to have quickly demasked the word (by simply hitting the space bar four or five times in a row) without even trying to identify the word at an earlier level within the sequence of fragmentations. Once they reached a sufficiently complete version of the word (about Level 4 or 5), they seem to have guessed a word that is compatible with the visual information. This particular type of guessing strategy-rapid demasking until Level 4 or 5 and immediate guessing, even if the resolution of potential ambiguity with respect to multiple word candidates would require that one go on to a less fragmented levelefficiently reduces the time per trial. There are two indicators in favor of this interpretation. First, Subjects 8 to 10 rarely responded before Level 4 or after Level 6 . Second, the experimenter reported that the time they took to go from fragmentation Level 1 to Level 4 was much shorter than that of Subjects $1-7$, which indicates that they did not fully explore the visual input during early levels of fragmentation.

We attempted to tune our simulation model to capture these different response patterns. Two parameters of the original interactive activation made it possible to generalize the model so as to predict individual performance in the fragmentation task: word-letter feedback and letter-word inhibition. Word-letter feedback would reflect the subjects' ability to exploit their lexical knowledge to mentally fill in the missing fragments. Variations in this parameter would therefore reflect lexical top-down influences on perception. Letter-word inhibition, on the other hand, determines the extent to which word units are inhibited when one or more letter units are incompatible with it. Variations in this parameter would then reflect the subjects' ability to pay close attention to the visual input and to exclude those word candidates from consideration that are not compatible with the fragmented input.

As can be seen in Figure 2B, we were able to simulate the speed-accuracy tradeoff obtained for Subjects 1-7 and the particular response strategy of Subjects $8-10$ by systematically manipulating word-letter feedback and letter-word inhibition. The values of these two parameters used to simulate the individual response patterns of the 10 subjects are given in Table 2 .

As can be seen in Table 2, the speed-accuracy tradeoff of Subjects 1-7 is simulated by an inverse manipulation of word-letter feedback and letter-word inhibition. Subjects with a conservative response strategy (Subjects $1-4)$ are simulated with a high letter-word inhibition and a low word-letter feedback. High letter-word inhibition pulls down the activation of incompatible word candidates, which reduces the possibility of errors. Low word-letter feedback reduces the overall lexical activity, which increases identification thresholds (fragmentation levels). These parameter values change inversely as subjects trade levels against errors. For example, the opposite response pattern of a subject using a guessing strategy (Subject 7) is simulated with a low letter-word inhibition (which increases the possibility of errors) and a high word-letter feedback (which reduces fragmentation lev-

Table 2

Parameter Values (Word-Letter Feedback and Letter-Word

Inhibition) Used to Simulate Individual Response Patterns

\begin{tabular}{ccc}
\hline & \multicolumn{2}{c}{ Parameter } \\
\cline { 2 - 3 } Subjects & $\begin{array}{c}\text { Word-Letter } \\
\text { Feedback }\end{array}$ & $\begin{array}{c}\text { Letter-Word } \\
\text { Inhibition }\end{array}$ \\
\hline 1 & .070 & .022 \\
2 & .085 & .022 \\
3 & .080 & .022 \\
4 & .080 & .022 \\
5 & .110 & .022 \\
6 & .120 & .020 \\
7 & .140 & .018 \\
8 & .080 & .010 \\
9 & .087 & .015 \\
10 & .090 & .010 \\
\hline
\end{tabular}


els). The performance of Subjects 8-10 (late and errorprone responding) is simulated with a much lower letterword inhibition and a relatively low word-letter feedback.

In sum, the model does a fairly good job in predicting the individual tradeoff between error rates and identification thresholds. This tradeoff is simulated by an inverse and systematic manipulation of letter-word inhibition and word-letter feedback. Increasing letter-word inhibition may be interpreted as an attentional bottom-up parameter that reduces the model's error rate. Increasing wordletter feedback may be interpreted as a lexical top-down parameter that reduces simulated identification thresholds (fragmentation levels).

\section{Model Evaluation}

In this section, we evaluate the model's ability to account for the effects of those variables that exhibited the strongest correlation with performance in the fragmentation task. These variables are log word frequency on identification thresholds $\left(r_{\text {partial }}=-.313\right)$, neighborhood size on errors $\left(r_{\text {partial }}=.164\right)$, and letter confusability on identification thresholds $\left(r_{\text {partial }}=.449\right)$. Figure 3 compares the subjects' with the model's performance for these three effects. Note that we presented all 580 words 10 times to the model ( 10 simulated subjects). Therefore, both the obtained and simulated effects are based on a large number of data points.

Figure $3 \mathrm{~A}$ shows the classic word frequency effect: the higher a word's frequency of occurrence, the earlier it can correctly be identified in the sequence of demasking. In other words, high-frequency words can correctly be identified when only a few fragments of the word are visible. The interactive activation model captures the systematic decrease of identification levels with increasing word frequency. However, the model overestimates the size of the effect. On the one hand, low-frequency words are identified later by the model than by subjects. On the other hand, high-frequency words are identified earlier by the model than by subjects.

In the interactive activation model, word frequency is represented in terms of resting level activation of the word units. High-frequency words have a higher resting level activation than do low-frequency words. They benefit from a head start as compared with low-frequency words. We suppose that the interactive activation model overestimates the size of the frequency effect because the range of resting level activation is too wide. Narrowing the range of resting level activations could be considered as a minor technical modification that probably does not affect the model's ability to capture the highly systematic influence of word frequency on identification thresholds.

Figure 3B shows an inhibitory effect of neighborhood size on the error data. Subjects' error rates increase monotonically with the number of a word's orthographic neighbors. Words with many orthographic neighbors generate up to $15 \%$ errors. The interactive activation model captures well the monotonic increase of the error rate as a function of neighborhood size. However, as with the fre-
A

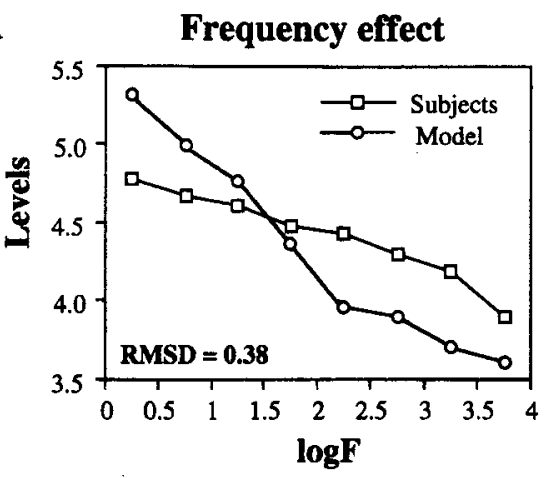

$\mathbf{B}$

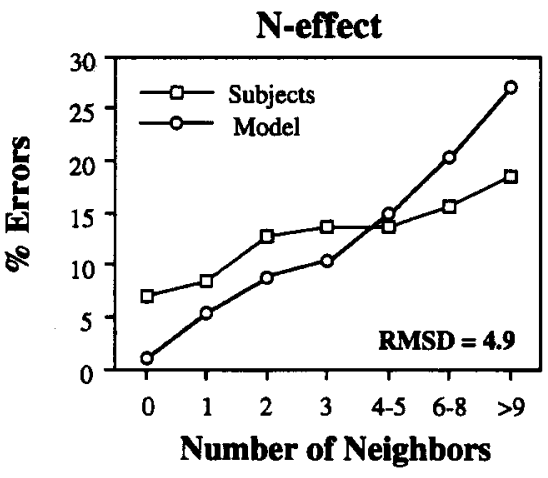

C
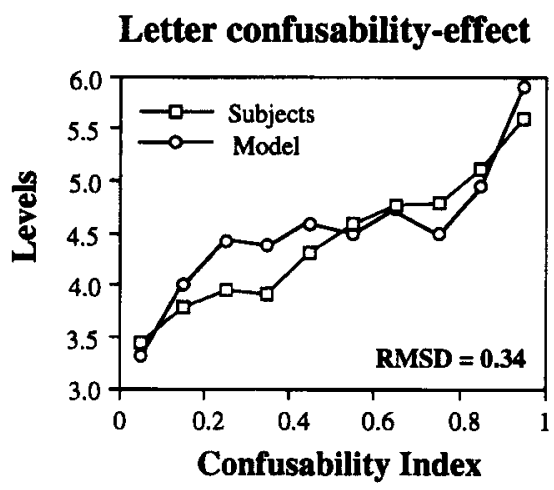

Figure 3. Major effects in the fragmentation task. Panel $A$ shows the frequency effect on identification thresholds; panel B, the neighborhood size effect on errors; and panel $C$, the confusability effect on identification thresholds. The model's fit is indicated in terms of root mean square deviations (RMSD). RMSD gives the average square deviation between the predicted and observed values.

quency effect, the model overestimates the size of the neighborhood effect. With respect to words with only few neighbors, the model makes fewer errors than do subjects. It is possible that this failure is related to the size of the implemented lexicon (580 words). Since errors are generated on the basis of the implemented lexicon, the model makes fewer errors than subjects do because it "knows" fewer words than subjects do. An examination of the type of errors produced by the subjects supports this interpretation: $30 \%$ of the incorrect word responses produced by the subjects were words that are not in the model's imple- 
mented lexicon, such as very low-frequency words, conjugated verbs, or the plural form of nouns. However, this finding cannot explain why the model makes more errors than do subjects for words with many neighbors.

It is also possible that the model overestimates the size of the neighborhood effect because its simple structure fails to account for linguistic variables other than word frequency, orthographic neighborhood, or confusability. For example, in the model, words with no neighbors, socalled hermits, produce virtually no errors. Hermits benefit from the virtual absence of lexical competition (lexical inhibition) because they have no neighbors. Thus, the model makes the strong prediction that hermits should be the most easily identifiable words. However, since hermits often contain unique spellings and inconsistent sound-to-spelling correspondences (see Ziegler, Jacobs, \& Stone, 1996; Ziegler, Stone, \& Jacobs, 1997), they have been shown to produce inflated RTs and error rates in the lexical decision task (Waters \& Seidenberg, 1985; Ziegler, Montant, \& Jacobs, 1997). Thus, the present version of the model may overestimate the neighborhood size effect because it does not account for variables that go beyond purely orthographic and lexical processing, such as variables related to phonological processes in word recognition (see, e.g., Berent \& Perfetti, 1995; Van Orden, Pennington, \& Stone, 1990; Ziegler \& Jacobs, 1995; Ziegler, Van Orden, \& Jacobs, 1997; for an extension of the interactive activation model that includes phonological processes, see Jacobs, Rey, Ziegler, \& Grainger, in press).

Figure $3 \mathrm{C}$ illustrates the effect of letter confusability on identification thresholds. The higher the confusability of a word's constituent letters, the more demasking is needed to correctly identify the word. Considering the history of the interactive activation model, it is interesting that the model captures the systematic bottom-up effect of letter confusability. Originally, this model was developed to capture context (top-down) effects on letter perception. However, as the present simulation shows, it also does a good job in dealing with bottom-up effects, such as letter confusability.

Note that the present simulation results concerning the effects of frequency, neighborhood size, and confusability were not obtained from different simulations. They are simply different illustrations of the model's behavior (errors and identification thresholds) for the 580 words that we presented to the model. Thus, one and the same simulation captures the facilitatory effects of frequency on identification thresholds, the inhibitory neighborhood size effects on errors, and the inhibitory letter confusability effects on thresholds.

\section{GENERAL DISCUSSION}

In the present article, we collected word identification thresholds and errors in Snodgrass's fragmentation task for all four-letter French words. In the first part of this article, we attempted to find out which of the standard variables known to affect word recognition performance in other tasks covary with performance measures in the fragmentation task. In the second part, we analyzed individual response performance and identified different response strategies. In a further step, we demonstrated that the interactive activation model can account for those individual response strategies by adapting two parameters: word-letter feedback and letter-word inhibition. In the third part, we demonstrated that the adaptation of the interactive activation model to the fragmentation task made it possible to successfully simulate a facilitatory frequency effect on identification thresholds, an inhibitory neighborhood size effect on error rates, and an inhibitory letter confusability effect on identification thresholds. When the task-specific processes of the fragmentation task are specified and the subject-specific response strategies are included, the interactive activation model provides a parsimonious architecture for modeling the taskindependent processes involved in word perception.

\section{Which Variables Affect Performance in the Fragmentation Task: Top-Down and Bottom-Up Effects on Perception}

As concerns the variables that affect performance in the fragmentation task, the regression analyses suggest the following global picture: Word frequency and confusability affect word identification thresholds, whereas neighborhood structure and orthographic redundancy affect error rates. This global picture contains a number of interesting details. First, it is logarithmic word frequency rather than plain frequency that constitutes the better predictor for identification thresholds (for a discussion of this issue, see Massaro \& Cohen, 1994; Paap \& Johansen, 1994).

Second, letter confusability, as estimated in a separate letter identification study (Rey et al., 1996), constitutes a powerful predictor of word identification thresholds. The more confusable the letters, the harder it is to identify the word (cf. Rumelhart \& Siple, 1974). Note that, although all of the independent variables that are based on lexical statistics (frequency, neighborhood structure, and orthographic redundancy) are highly intercorrelated, none of them is correlated with letter confusability. Therefore, letter confusability reflects the visual, bottom-up information that is independent of the lexical, top-down information, as measured by such variables as frequency and neighborhood structure. These results neatly show that both bottom-up and top-down information jointly influence word perception in the fragmentation task. Future research could try to investigate the tradeoff between bottom-up and top-down information by quantifying these contributions across different tasks and different subjects.

Third, it seems that different classes of independent variables predict identification thresholds and error rates in the fragmentation task. Word frequency affects identification thresholds but not error rates. Neighborhood structure affects error rates but not identification thresholds. Note that positional letter frequency also predicts 
error rates. However, positional letter frequency and neighborhood size are highly correlated (.546) because of the fact that, the more neighbors a word has, the higher the probability that a letter at a given position occurs in all of its neighbors. Since it seems that neighborhood size and positional letter frequency essentially measure similar things, as can be seen by the reduction of the partial correlations of both variables when the variance explained by the other variable is pulled out, we do not consider them separately here. Finally, letter confusability affects both identification thresholds and error rates.

The dissociation between variables that affect identification thresholds (e.g., frequency) and variables that affect errors (e.g., neighborhood structure) can be described by borrowing the language of dynamic systems theory in psychology (Elman, 1995; Kawamoto, 1993; Kawamoto \& Zemblidge, 1992; Stone \& Van Orden, 1994; Van Orden \& Goldinger, 1994). Performance in the fragmentation task may be thought of as a trajectory through the linguistic state space from the initial encodings to an attractor point. Each attractor in state space is bounded by a separatrix, a (high-dimensional) boundary that circumscribes an attractor basin. Within the attractor basin, the initial encodings are moved toward the respective attractor point. Beyond this boundary, encodings fall in the basin of some other attractor (see Van Orden \& Goldinger, 1994). Identification thresholds in the fragmentation task may be a function of the dynamics that move encodings toward the respective attractor point. The gradient of this trajectory seems to be affected by a word's frequency. However, errors may reflect encodings that fall beyond the correct attractor basin into the basin of an adjoining attractor. Orthographic neighborhood seems to be one of the variables that determine the number and the overlap of adjacent attractor basins.

\section{Simulating Individual Differences}

In the present article, we followed an experimental approach to studying word perception that shares aspects with a psychophysical approach: many trials and few subjects (cf. Rumelhart \& Siple, 1974). In contrast, the typical psycholinguistic approach uses a few critical trials and a large number of subjects. One of the advantages of the psychophysical approach is that it gives a stable estimate of individual response patterns, since it allows subjects to adapt their response strategy throughout a large number of trials. In fact, among the 10 subjects tested in this experiment, we found quite distinct response patterns. Seven subjects showed a remarkable speedaccuracy tradeoff. Their adaptation to the task consisted in trading speed (levels) against accuracy (errors). The extreme on one side was a conservative response strategy; the extreme on the other side was a guessing strategy. One group of subjects showed a very particular response strategy. This strategy consisted in (1) quickly passing on to a less fragmented version of the word without any exploration of the visual input at earlier levels of fragmentation and (2) guessing a word on the basis of this less fragmented input, even if the visual information was not sufficient to decide between competing word candidates.

The interactive activation model provides a parsimonious, elegant, and transparent way of simulating individual response strategies in the fragmentation task by adapting two parameters: letter-word inhibition and wordletter feedback. These parameters can psychologically be interpreted in the following way: Letter-word inhibition could be assimilated to the concept of attention. It may reflect the subject's ability to detect a visual incompatibility between the fragmented letter information and the potential word candidates that come to mind. For example, subjects may perceive at the initial position of the word a letter that looks like an F or a P. However, if they notice in the first letter position a pixel in the right bottom corner of the array, then they know that the first letter cannot be $F$ or $P$, and they can exclude all words that start with an F or a P, even if they initially thought that the word must start with an F or a P. This is exactly the function of letter-word inhibition in the model. It inhibits word units that are incompatible with the letter information. As the individual simulations have shown, an increase in this parameter effectively reduces the model's error rate.

On the other hand, word-letter feedback could reflect subjects' ability to exploit lexical and spelling knowledge to mentally fill in the missing fragments. In the model, increasing this parameter increases the overall activation (resonance) between compatible letter and word units. Increasing this parameter therefore reduces the time it takes for the model to identify a word (i.e., the simulated fragmentation levels).

\section{Task-Specific Modeling}

In this article, we demonstrated how the interactive activation model that typically simulates the automatic and fast processes of word perception (Jacobs \& Grainger, 1992) can be extended to simulate word identification thresholds and errors in the fragmentation task. To this end, we specified and implemented the task-specific mechanisms that made it possible to connect the internal mechanisms of the model (interactive activation, competition, intralevel inhibition) to observable, task-specific, strategic, and individual performance, as measured by two independent variables. The model succeeded in simulating individual performance and the graded nature of a variety of effects obtained in this task.

One of the interesting results of the regression analysis was that lexical, top-down factors (frequency and neighborhood structure) and visual, bottom-up factors (letter confusability) provided strong and independent contributions to predicting performance in the fragmentation task. The simulation results show that the model does a good job in accounting for the lexical, top-down effects on fragmentation performance. In some ways, this is less surprising, since the model was originally designed to capture context effects on letter and word perception (i.e., the word superiority effect). However, as 
the present simulation results show, the model also nicely predicts the bottom-up effects of letter confusability. The relatively simple structure of the interactive activation model (feature, letter, and word levels) allows it to capture some of the joint influence of visual bottom-up information and lexical top-down information on perception. In particular, our simulations show that the model, like the subjects, is able to trade bottom-up information against top-down information. Thus, the principles of interactive activation and the present task-specific implementation seem to provide a parsimonious description of the fluid coupling of subject and laboratory method.

\section{REFERENCES}

ANDREWs, S. (1989). Frequency and neighborhood size effects on lexical access: Activation or search? Journal of Experimental Psychology: Learning, Memory, \& Cognition, 15, 802-814.

ANDREws, S. (1992). Frequency and neighborhood effects on lexical access: Lexical similarity or orthographic redundancy? Journal of Experimental Psychology: Learning, Memory, \& Cognition, 18, 234-254.

Berent, I., \& Perfetti, C. A. (1995). A rose is a reez: The two-cycles model of phonology assembly in reading English. Psychological Review, 102, 146-184.

Brown, P., LupKer, S. J., \& Colombo, L. (1994). Interacting sources of information in word naming: A study of individual differences. Journal of Experimental Psychology: Human Perception \& Performance, 20, 537-554.

CaramazzA, A. (1992). Is cognitive neuropsychology possible? Journal of Cognitive Neuropsychology, 4, 80-95.

Coltheart, M., Davelaar, E., Jonasson, J. T., \& Besner, D. (1977) Access to the internal lexicon. In S. Dornic (Ed.), Attention and performance VI (pp. 535-555). London: Academic Press.

CUTLER, A. (1981). Making up materials is a confounded nuisance, or: Will we be able to run any psycholinguistic experiments at all in 1990? Cognition, 10, 65-70.

DELL, G. S. (1986). A spreading activation theory of retrieval in sentence production. Psychological Review, 93, 405-434.

Elman, J. L. (1995). Language as a dynamical system. In R. F. Port \& T. van Gelder (Eds.), Mind as motion (pp. 196-225). Cambridge, MA: MIT Press.

Forster, K. I., \& ChAmbers, S. M. (1973). Lexical access and naming time. Journal of Verbal Learning \& Verbal Behaviour, 12, 627-635.

Frauenfelder, U., BaAyen, R. H., Hellwig, F. M., \& Schreuder, R. (1993). Neighborhood density and frequency across languages and modalities. Journal of Memory \& Language, 32, 781-804.

GRAINGER, J., \& JACOBS, A. M. (1993). Masked partial-word priming in visual word recognition: Effects of positional letter frequency. Journal of Experimental Psychology: Human Perception \& Performance, 19, 951-964.

Grainger, J., \& JaCOBS, A. M. (1994). A dual read-out model of word context effects in letter perception: Further considerations of the word superiority effect. Journal of Experimental Psychology: Human Perception \& Performance, 20, 1158-1176.

Grainger, J., \& JACOBS, A. M. (1996). Orthographic processing in visual word recognition: A multiple read-out model. Psychological Review, 103, 518-565.

Grainger, J., O'Regan, J. K., Jacobs, A. M., \& Segui, J. (1989). On the role of competing word units in visual word recognition: The neighborhood frequency effect. Perception \& Psychophysics, 45, 189-195.

Grainger, J., O'Regan, J. K., Jacobs, A. M., \& Segui, J. (1992). Neighborhood frequency effects and letter visibility in visual word recognition. Perception \& Psychophysics, 51, 49-56.

HunTSMan, L. A., \& LiMa, S. D. (1996). Orthographic neighborhood structure and lexical access. Journal of Psycholinguistic Research, 25, 417-429.

IMBs, P. (1971). Études statistiques sur le vocabulaire français: Diction- naire des fréquences. Vocabulaire littéraire des XIXe et XXe siècles [Statistical analysis of French vocabulary: Frequency dictionary. Vocabulary from the 19th and 20th centuries]. Paris: Librairie Marcel Didier.

JaCobs, A. M., \& GRainger, J. (1992). Testing a semi-stochastic variant of the interactive activation model in different word recognition experiments. Journal of Experimental Psychology: Human Perception \& Performance, 18, 1174-1188.

Jacobs, A. M., Rey, A., Ziegler, J. C., \& Grainger, J. (in press). MROM-P: An interactive activation, multiple read-out model of orthographic and phonological processes in visual word recognition. In J. Grainger \& A. M. Jacobs (Eds.), Symbolic connectionist approaches to human cognition. Hillsdale, NJ: Erlbaum.

KaWAMOTO, A. H. (1993). Nonlinear dynamics in the resolution of lexical ambiguity: A parallel distributed processing account. Journal of Memory \& Language, 32, 474-516.

Kawamoto, A. H., \& Zemblidge, J. (1992). Pronunciation of homographs. Journal of Memory \& Language, 31, 349-374

LANDAUER, T. K., \& STREeTER, L. A. (1973). Structural differences between common and rare words: Failure of equivalence assumptions for theories of word recognition. Journal of Verbal Learning \& Verbal Behaviour, 12, 119-131.

Lemaire, P., \& SiEgLER, R. S. (1995). Four aspects of strategic change: Contributions to children's learning of multiplication. Journal of Experimental Psychology: General, 124, 83-97.

MASSARO, D. W., \& COHEN, M. M. (1994). Visual, orthographic, phonological, and lexical influences in reading. Journal of Experimental Psychology: Human Perception \& Performance, 20, $1107-1128$.

Massaro, D. W., Jastrzembski, J. E., \& Lucas, P. A. (1981). Frequency, orthographic regularity, and lexical status in letter and word perception. In G. H. Bower (Ed.), The psychology of learning and motivation (Vol. 15, pp. 163-200). New York: Academic Press.

McClelland, J. L., \& RumelHarT, D. E. (1981). An interactive activation model of context effects in letter perception: Part I. An account of basic findings. Psychological Review, 88, 375-407.

MONSELL, S. (1991). The nature and locus of word frequency effects in reading. In D. Besner \& G. W. Humphreys (Eds.), Basic processes in reading: Visual word recognition (pp. 148-197). Hillsdale, NJ: Erlbaum.

PAAP, K., \& JohANSEN, L. (1994). The case of the vanishing frequency effect: A retest of the verification model. Journal of Experimental Psychology: Human Perception \& Performance, 6, 1129-1157.

Peereman, R., \& Content, A. (1995). The neighborhood size effect in naming: Lexical activation or sublexical correspondences. Journal of Experimental Psychology: Learning, Memory, \& Cognition, 21, 409-421.

REDER, L. (1987). Strategy selection in question answering. Cognitive Psychology, 19, 90-137.

REDER, L. (1988). Strategic control of retrieval strategies. In G. Bower (Ed.), The psychology of learning and motivation (Vol. 22, pp. 227259). New York: Academic Press.

Rey, A., Ziegler, J. C., Montant, M., \& Jacobs, A. M. (1996, September). Static and dynamic approaches to letter perception. Paper presented at the 9th Conference of the European Society for Cognitive Psychology, Würzburg.

Rumelhart, D. E., \& McClelland, J. L. (1982). An interactive activation model of context effects in letter perception: Part II. The contextual enhancement effect and some tests and extensions of the model. Psychological Review, 89, 60-94.

RumelharT, D. E., \& Siple, P. (1974). The process of recognizing tachistoscopically presented words. Psychological Review, 81, 99-118.

Schade, U., \& Berg, T. (1992). The role of inhibition in a spreadingactivation model of language production: Part 2 . The simulation perspective. Journal of Psycholinguistic Research, 21, 435-462.

SeARS, C. R., Hino, Y., \& LuPKER, S. J. (1995). Neighborhood size and neighborhood frequency effects in word recognition. Journal of Experimental Psychology: Human Perception \& Performance, 21, 876-900.

SIEGLER, R. S. (1987). The perils of averaging data over strategies: An example from children's addition. Journal of Experimental Psychology: General, 106, 250-264.

SnODGRASs, J. G., \& Mintzer, M. (1993). Neighborhood effects in vi- 
sual word recognition: Facilitatory or inhibitory? Memory \& Cognition, 21, 247-266.

Snodgrass, J. G., \& Poster, M. (1992). Visual-word recognition thresholds for screen-fragmented names of the Snodgrass and Vanderwart pictures. Behavior Research Methods, Instruments, \& Computers, 24, 1-15.

SPERLING, G., \& Dosher, B. A. (1986). Strategy and optimization in human information processing. In K. R. Boff, L. Kaufman, \& J. P Thomas (Eds.), Handbook of perception and human performance (pp. 2.4-2.65). New York: Wiley.

SPIEler, D. H., \& Balota, D. A. (1996, November). Current connectionist models of word naming: What they can and cannot do. Paper presented at the 37th Annual Meeting of the Psychonomic Society, Chicago.

Stone, G. O., \& Van Orden, G. C. (1993). Strategic control of processing in word recognition. Journal of Experimental Psychology: Human Perception \& Performance, 19, 744-774.

StONe, G. O., \& VAN ORDEN, G. C. (1994). Building a resonance framework for word recognition using design and system principles. Journal of Experimental Psychology: Human Perception \& Performance, 20, 1248-1268.

Treiman, R., Mullennix, J., Bijeljac-Babic, R., \& RichmondWELTY, E. D. (1995). The special role of rimes in the description, use, and acquisition of English orthography. Journal of Experimental Psychology: General, 124, 107-136.

UNDERWOOD, B. J. (1975). Individual differences as a crucible in theory construction. American Psychologist, 30, 128-134.

Van Orden, G. C., \& Goldinger, S. D. (1994). Interdependence of form and function in cognitive systems explains perception of printed words. Journal of Experimental Psychology: Human Perception \& Performance, 20, 1269-1291.

Van Orden, G. C., Pennington, B. F., \& Stone, G. O. (1990). Word identification in reading and the promise of subsymbolic psycholinguistics. Psychological Review, 97, 488-522.

WarRington, E. K., \& WeISKRaNTZ, L. (1968). New method of testing long-term retention with special reference to amnesic patients. Nature, 217, 972-974

WATERS, G. S., \& SEIDENBERG, M. S. (1985). Spelling-sound effects in reading: Time course and decision criteria. Memory \& Cognition, 13 , 557-572.

Ziegler, J. C., \& JACOBS, A. M. (1995). Phonological information provides early sources of constraint in the processing of letter strings. Journal of Memory \& Language, 34, 567-593.

Ziegler, J. C., JACOBS, A. M., \& STONE, G. O. (1996). Statistical analysis of the bidirectional inconsistency of spelling and sound in French Behavior Research Methods, Instruments, \& Computers, 28, 504-515.

Ziegler, J. C., Montant, M., \& JacoBs, A. M. (1997). The feedback consistency effect in French. Journal of Memory \& Language, 37, 533-554.

Ziegler, J. C., Stone, G. O., \& JACOBS, A. M. (1997). What's the pronunciation for _ough and the spelling for $/ \mathrm{u} /$ ? A database for computing feedforward and feedback inconsistency in English. Behavior Research Methods, Instruments, \& Computers, 29, 600-618.

ZiegleR, J. C., VAN ORDEN, G. C., \& JaCOBS, A. M. (1997). Phonology can help or hurt the perception of print. Journal of Experimental Psychology: Human Perception \& Performance, 23, 845-860.

\section{NOTE}

1. We are grateful to Gay Snodgrass for suggesting this analysis.

(Manuscript received September 17, 1995; revision accepted for publication December 24,1996 .) 